\title{
Estrutura e regeneração de Bertholletia excelsa Bonpl. em castanhais nativos da Amazônia
}

\author{
Structure and regeneration of Bertholletia excelsa Bonpl. in Brazil nut groves in \\ the Amazon
}

\author{
Helio Tonini ${ }^{\mathrm{I}}$, Aisy Botega Baldoni ${ }^{\mathrm{II}}$
}

\begin{abstract}
Resumo
O trabalho foi desenvolvido com o objetivo de comparar a estrutura diamétrica e os indicadores de regeneração natural em castanhais nativos e correlacioná-los à fase de desenvolvimento da floresta, à intensidade de coleta de sementes e à exploração madeireira. Os dados foram coletados em 54 hectares nos municípios de Caracaraí - RR e Itaúba - MT. As distribuições de frequência diamétricas foram comparadas por medidas de tendência central, variabilidade, forma e aderência por Kolmogorov-Smirnov. A regeneração natural foi correlacionada às variáveis ecológicas, intensidade de coleta e à atividade da fauna pelo Coeficiente de Correlação de Spearman e as medias comparadas por Kruskal-Wallis. Os castanhais apresentaram estruturas diamétricas diferenciadas, o que pode ser atribuído a diferenças no percentual de indivíduos jovens nas menores classes diamétricas. A maior densidade de regenerantes nas clareiras e a inexistência de correlação significativa entre a taxa de coleta e os indicadores de regeneração natural indicaram que a abertura do dossel provocada pela exploração florestal madeireira pode ter sido o fator de maior impacto no estabelecimento da regeneração natural e no rejuvenescimento dos castanhais.

Palavras-chave: Castanheira-do-brasil; Mato Grosso; Roraima; Intensidade de coleta de sementes
\end{abstract}

\begin{abstract}
This work was developed with the objective of comparing the diameter structure and natural regeneration indicators of Brazil nut groves and correlates them to the forest successional stage, seed collection intensity and logging. Data were collected from 54 hectares in Caracaraí (Roraima state) and Itaúba (Mato Grosso state). Frequency distributions were compared by measures of central tendency, variability, form and adherence by Kolmogorov-Smirnov Test. Natural regeneration was correlated with environmental variables, seed collection intensity and fauna activity by Spearman correlation coefficient and KruskalWallis. The Brazil nut groves presented different diametric structures, which can be attributed to differences in young individuals in the smaller diametric classes. The higher density of juveniles in canopy gaps and the lack of a significant correlation between seed collection intensity and natural regeneration indicate that canopy openings from logging could have been the major impact factor in the natural regeneration establishment and in Brazil nut grove rejuvenation.
\end{abstract}

Keywords: Brazil nut; Mato Grosso; Roraima; Seed collection intensity

Engenheiro Florestal, Dr., Pesquisador da Embrapa Pecuária Sul, Rodovia BR-153, Km 632,9, Vila Industrial, Zona Rural, Caixa Postal 242, CEP 96401-970, Bagé (RS), Brasil. helio.tonini@embrapa.br (ORCID: 0000-0003-1123-7604)

II Agrônoma, Dra., Pesquisadora da Embrapa Agrossilvipastoril, Rod. dos Pioneiros, MT-222, Km 2,5, Zona Rural, Caixa Postal: 343 CEP 78550-970, Sinop (MT), Brasil. aisy.baldoni@embrapa.br (ORCID:0000-0002-8355-6957) 


\section{Introdução}

Dentre os produtos florestais não madeireiros da Amazônia, as amêndoas da castanheirado-brasil (Bertholletia excelsa, Bonp.) é um dos mais conhecidos e solidamente estabelecidos no mercado nacional e de exportação.

A alta densidade e o padrão de distribuição espacial agregado das árvores facilitam a atividade extrativista (WADT; KAINER; GOMES-SILVA, 2005). Estudos recentes afirmam que esta característica pode ter origem antropogênica, resultante de plantios e/ou condução da regeneração natural no sistema de cultivo do tipo derruba-e-queima, praticado por indígenas no passado (CLEMENT et al., 2010; SHEPARD JUNIOR; RAMIREZ, 2011; TOMAS et al., 2015) e também como o resultado do padrão de dispersão a curtas distâncias por roedores caviomorfos do gênero Dasyprocta sp. (TUCKHAUGAASSEN et al., 2010).

A baixa regeneração sob dossel fechado estimulou o surgimento da hipótese de que as populações não regeneravam e estavam sob ameaça de extinção. Ao verificarem esta hipótese, (PERES et al., 2003) concluíram que os níveis de coleta de sementes teriam grande impacto no recrutamento e na sustentabilidade da atividade extrativista a longo prazo, o que poderia levar os castanhais a um colapso demográfico.

Outra característica observada em castanhais nativos é a predominância de árvores em classes diamétricas intermediárias, que além da hipótese antropogênica, também é explicada por teorias como a das sementes como gargalo (ocasionado pela escassez severa de sementes decorrente de altos níveis de extração); a do gargalo mutualístico (que relaciona a ausência de regeneração natural a níveis insuficientes de sementes e de polinizadores), e a do gargalo para a viabilidade da regeneração (que relaciona a sua ausência a inexistência de clareiras na floresta), já que a espécie se estabelece e cresce melhor em grandes clareiras durante os estágios iniciais (SALOMÃO, 1991; PERES; BAIDER, 1997; ZUIDEMA; BOOT, 2002; ZUIDEMA, 2003).

Com base nas conclusões de Peres et al. (2003), foram feitas recomendações para viabilizar a regeneração natural em florestas com dossel fechado sujeitas à exploração intensiva de sementes, como o estabelecimento de cotas anuais de coleta, plantios de enriquecimento, restrições à caça e rotação de áreas (talhões) com a manutenção de compartimentos não explorados (MYERS; NEWTON; MELGAREJO, 2000; PEÑA-CLAROS et al., 2002; PERES et al., 2003; CONKLETON; GUARIQUATA; ALBORNOZ, 2011).

Porém, as conclusões de Peres et al. (2003) têm sido refutadas por autores como Wadt et al. (2008), Paiva, Quedes e Funi (2011) e Scoles e Gribel (2012; 2015), que têm observado que a dispersão de sementes não intencional realizada pelos extrativistas durante as etapas de coleta, transporte e armazenamento de sementes e que os distúrbios no dossel praticados por atividades humanas como a limpeza da base das castanheiras e a aberturas de picadas e o corte seletivo de madeira têm maior importância na manutenção e no rejuvenescimento dos castanhais do que a taxa de coleta de frutos e sementes (SCOLES; GRIBEL, 2012; 2015; SOUZA; ALMEIDA; AMARAL, 2014).

Uma vez que o clima de luz ou a abertura do dossel parece ser o principal fator ecológico na dinâmica de regeneração da castanheira-do-brasil (MYERS; NEWTON; MELGAREJO, 2000, PEÑA-CLAROS et al., 2002; SCOLES; KLEIN; GRIBEL, 2014) atividades que provoquem grandes alterações no dossel como a exploração madeireira devem induzir a regeneração com mais eficiência do que restrições ou cotas de coleta de frutos.

Neste contexto, este trabalho foi desenvolvido com o objetivo de comparar a estrutura diamétrica das árvores e os indicadores de regeneração natural em castanhais nativos e correlacioná-los à fase de desenvolvimento da floresta, a intensidade de coleta de sementes e a exploração madeireira. 


\section{Material e método}

\section{Características dos locais de estudo}

Os dados foram coletados nos municípios de Caracaraí - RR e Itaúba - MT, observados na Figura 1. Em Caracaraí, a floresta é do tipo Ombrófila Densa, com relevo plano a ondulado (BRASIL, 1975). Em Itaúba, a floresta é do tipo estacional semidecidual submontana com dossel emergente (IBGE, 2012).

O clima na região de Caracaraí é, segundo a classificação de Köppen, do tipo Af, tropical úmido ou superúmido, sem estação seca, com precipitação média anual entre 1700-2000 mm. Em Itaúba, o clima é do tipo Am tropical úmido ou subúmido, com precipitação média anual em torno de $2000 \mathrm{~mm}$ e período seco bem marcado, variando de dois a três meses (ALVARES et al., 2013; SOUZA; ALMEIDA; AMARAL, 2014).

\section{Figura 1 - Localização dos locais de coleta de dados na Amazônia Brasileira. ITA = região} do Itã (Caracaraí - RR); CUJ = Região do Cujubim (Caracaraí - RR) e ITB = Itaúba - MT.

Figure 1 - Location of data collection sites in the Brazilian Amazon ITA = Itã region (Caracarai, RR state); CUJ = Cujubim region (Caracarai, RR state) and ITB = Itaúba (MT state).

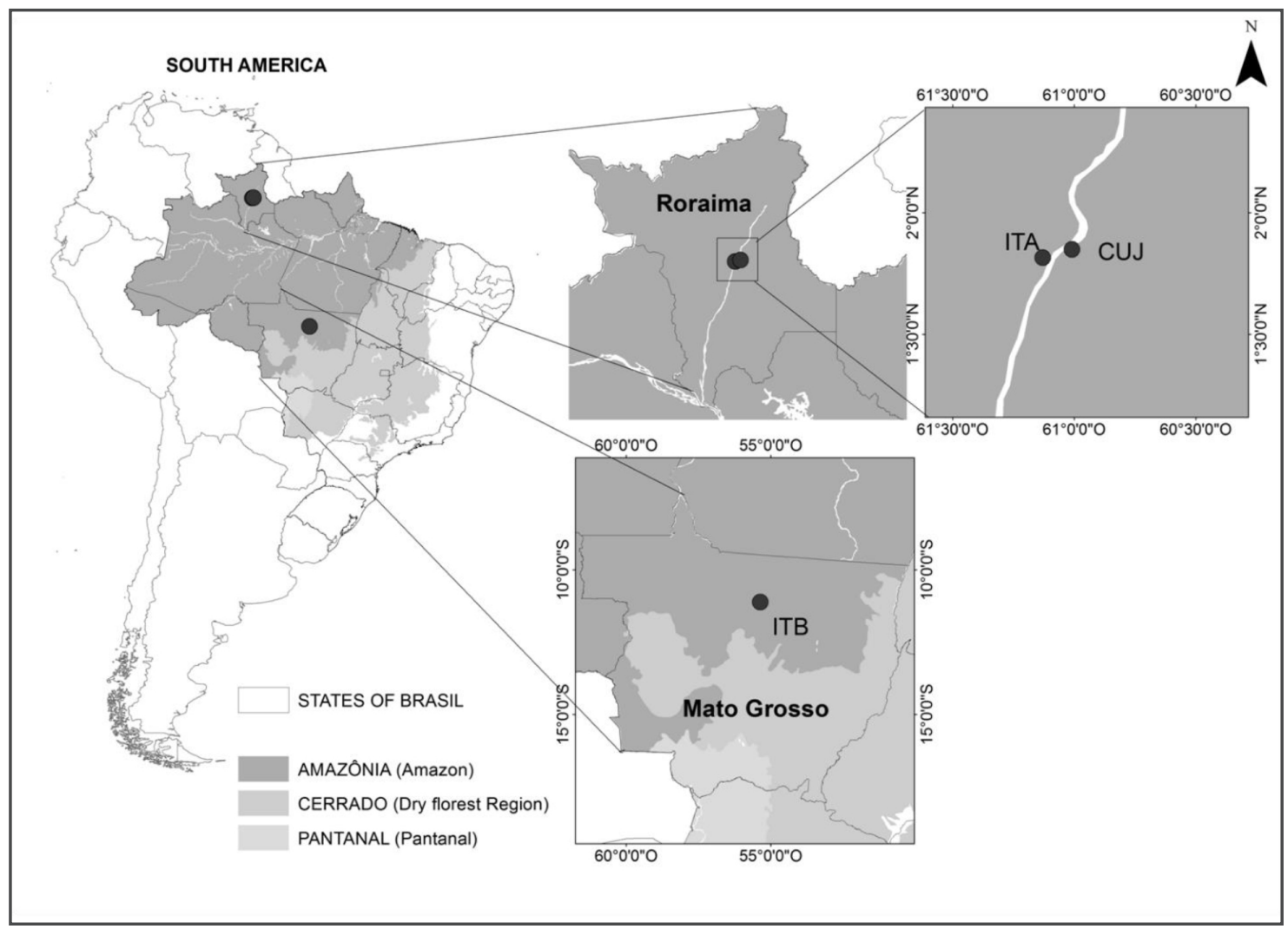

Fonte: Autores (2019)

Os locais estudados representam comunidades de fácil acesso rodoviário, submetidas ao extrativismo anual da castanha com e sem a extração madeireira recente. Em Caracaraí RR selecionaram-se dois castanhais, sendo o primeiro com área de aproximadamente 400 ha, localizado no projeto de assentamento do Itã (ITA) nas coordenadas $01^{\circ} 48^{\prime} 58^{\prime \prime}$ de latitude norte e $61^{\circ} 07^{\prime} 41^{\prime \prime}$ de longitude oeste, e o segundo em um projeto de manejo florestal madeireiro perten- 
tencente à Madeireira Vale Verde Ltda., localizado próximo ao projeto de assentamento Cujubim (CUJ), nas coordenadas $1^{0} 51^{\prime} 2,6^{\prime \prime}$ de latitude norte e $61^{\circ} 00^{\prime} 30,6^{\prime \prime}$ de longitude oeste. A área de manejo florestal está localizada na Fazenda Mundo com área total de 17.205,4 ha, explorados sob o sistema de impacto reduzido, com um ciclo de corte de 25 anos.

ITA representa uma comunidade não submetida à exploração madeireira recente, pois não existem registros desta atividade na área. Este castanhal está sujeito ao processo de fragmentação, pois é cercado por lotes de projeto de assentamento rural. Na área se pratica o extrativismo da castanha com a remoção de todos os frutos em uma ou duas ocasiões durante o período de queda dos frutos.

CUJ representou uma comunidade submetida à exploração madeireira recente (dois anos), em que se pratica o extrativismo da castanha todos os anos. Neste local, o extrativismo é realizado por comunitários do entorno com permissão da empresa proprietária.

Em Itaúba - MT, a área de estudo foi a reserva legal da Fazenda Santo Ângelo pertencente ao Grupo Dalpai, com área de 2000 hectares (ITB), localizada nas coordenadas geográficas de $11^{0} 5,6^{\prime} 41^{\prime}$ de latitude sul e $55^{0} 21,2^{\prime} 42^{\prime}$ de longitude oeste. Esta área foi explorada sob plano de manejo florestal sustentado em 1998 com ciclo de corte previsto para 25 anos. Representa uma população explorada com fins madeireiros há 18 anos que, atualmente, está sob a coleta constante e anual de sementes de castanha-do-brasil. O extrativismo é praticado sob sistema de arrendamento, em que os extrativistas coletam todos os frutos em uma ou duas ocasiões durante o período de queda dos frutos e pagam um percentual que varia de $10 \%$ a $15 \%$ da produção ao proprietário da área.

\section{Coleta dos dados}

A coleta dos dados foi realizada em seis parcelas permanentes (PPs) de nove hectares cada (300 m x $300 \mathrm{~m}$ ), sendo duas por local, selecionadas em função da facilidade de acesso e densidade de castanheiras. Em ITA as PPs foram instaladas em 2006 e 2007 (ITA1 e ITA2) a uma distância de $500 \mathrm{~m}$ entre as mesmas. Em CUJ foram instaladas em fevereiro de 2009 (CUJ1e CUJ2) uma em frente à outra, separadas pela estrada principal do projeto de manejo florestal. Em Itaúba foram instaladas em 2011(ITB1) e 2015 (ITB2), a uma distância em linha reta de 3,5 km entre as mesmas.

Nas parcelas permanentes, todas as castanheiras com diâmetro a altura do peito (DAP) $\geq 10 \mathrm{~cm}$ foram identificadas, medidas e numeradas com placas de alumínio. A produção de frutos foi monitorada anualmente, em pelo menos duas ocasiões, pela contagem e a pesagem das sementes durante o período de queda dos frutos (período chuvoso), que em Caracaraí, ocorre de abril a julho e em Itaúba de outubro a fevereiro.

O inventário da regeneração natural foi realizado em 2009 em ITA e CUJ, 2014 em ITB1, e 2015 em ITB2. Foram instaladas 36 subparcelas de $25 \mathrm{~m}$ x $25 \mathrm{~m}$, distribuídas aleatoriamente dentro de cada parcela permanente de $300 \mathrm{~m}$ x $300 \mathrm{~m}$, totalizando 2,25 ha de amostragem por parcela permanente e 13,5 ha no total.

Em cada subparcela, a regeneração natural foi estratificada conforme Wadt et al. (2008) e Scoles e Gribel (2015), em plântulas (indivíduos com altura menor do que 1,5 m); varetas (indivíduos com altura maior do que $1,5 \mathrm{~m}$ e DAP menor do que $10 \mathrm{~cm}$ ); e indivíduos jovens ou não produtivos $(10 \mathrm{~cm} \leq \mathrm{DAP} \leq 40 \mathrm{~cm})$. Em cada subparcela foram contados os frutos intactos e abertos por roedores e, desta forma, foi possível calcular a intensidade de coleta e a atividade da fauna, conforme Wadt et al. (2008).

A intensidade de coleta de frutos (IC) foi calculada por:

$$
I C \%=\left(\frac{N F c}{N F_{c}+N F_{n c}+N F_{a}}\right) x 100
$$


A atividade da fauna (AF) foi calculada por:

$$
A F \%=\left(\frac{N F_{a}}{N F_{n c}+N F_{a}}\right) x 100
$$

Em que: $\mathrm{NF}_{\mathrm{c}}=$ número de frutos coletados; $\mathrm{NF}_{\mathrm{nc}}=$ número de frutos não coletados e fechados; $\mathrm{NF}_{\mathrm{a}}=$ Número de frutos abertos por roedores.

Os inventários foram realizados durante a estação seca e cada unidade amostral foi classificada em relação à fase de desenvolvimento da floresta de acordo com a característica fisionômica que se encontrava no momento do levantamento, com base em Silva et al. (2005). Foram consideradas clareiras, as subparcelas com abertura de dossel de pelo menos $50 \%$ da área e poucas ou nenhuma árvore com $\mathrm{DAP} \geq 10 \mathrm{~cm}$; floresta em construção aquelas subparcelas que continham árvores com $10 \mathrm{~cm} \leq \mathrm{DAP} \leq 40 \mathrm{~cm}$ e a predominância de espécies pioneiras; floresta madura as que apresentaram a predominância de árvores de grandes dimensões (DAP $\geq 40 \mathrm{~cm}$ ) com pouca ou nenhuma abertura do dossel.

\section{Análise estatística}

As distribuições de frequência diamétricas foram comparadas em relação às medidas de tendência central (media e mediana), medidas de variabilidade (desvio padrão e variância), medidas de forma (assimetria e curtose) e pelo teste de aderência de Kolmogorov-Smirnov, a 5\% de probabilidade.

As plântulas, varetas e indivíduos jovens foram utilizados como indicadores da regeneração potencial, pré-estabelecida e estabelecida, respectivamente. Estes indicadores foram relacionados às variáveis ecológicas, como a fase de desenvolvimento da floresta, intensidade de coleta (IC\%), atividade da fauna (AF\%), produção média de sementes por ha (PS) e relação frutos não coletados por árvore adulta $\left(\mathrm{NF}_{\mathrm{nc}} / \mathrm{N}\right)$ pelo Coeficiente de Correlação de Spearman e teste de Kruskal-Walliss para um nível de significância de 5\%. Para esta análise também foram adicionados dados de estudos de mesma natureza como Wadt et al. (2008), Scoles e Gribel (2012; 2015) e Souza, Almeida e Amaral (2014).

\section{Resultados e discussão}

\section{Estrutura das populações}

As medidas de densidade, posição, dispersão, assimetria e curtose em relação aos diâmetros observados no tronco nas unidades amostrais (Tabela 2) indicaram que densidade das castanheiras $(\mathrm{DAP} \geq 10 \mathrm{~cm}$ ) variou de 6 a 15 indivíduos por hectare (Tabela 1), sendo maior nas parcelas localizadas em Itaúba. Esta densidade pode ser considerada média na comparação com as relatadas por Salomão (1991; 2009), Peres e Baider (1997), Peres et al. (2003), Wadt, Kainer e Gomes Silva (2005), Wadt et al. (2008), Scoles e Gribel (2012; 2015), Souza, Almeida e Amaral (2014), com variações entre 0,7 a 25 indivíduos ha-1 em diferentes locais da Amazônia Brasileira.

As populações apresentaram assimetria positiva, indicando maior concentração de indivíduos à esquerda das distribuições de frequência, ou seja, proporcionalmente, um maior número de indivíduos nas menores classes diamétricas. A população de Itaúba apresentou maior assimetria, indicando maior proporção de indivíduos jovens (Tabela 1). O grau de curtose indicou que em ITA a distribuição é mesocúrtica, em CUJ leptocúrtica e em ITB platicúrtica. 
Tabela 1 - Estatística descritiva para as populações de Bertholletia excelsa avaliadas na região do Itâ - RR (ITA), Cujubim - RR (CUJ) e Itaúba - MT (ITB).

Table 1 - Descriptive statistics for Bertholletia excelsa populations in the region of Itâ-RR state (ITA), Cujubim-RR state (CUJ) and Itaúba-MT state (ITB).

\begin{tabular}{ccccccccc}
\hline Local & G & N & \multicolumn{7}{c}{ DAP } & Assimetria & $\begin{array}{c}\text { Grau de } \\
\text { Curtose }\end{array}$ \\
\hline & & & Média & Mediana & Desvio padrão & Variância & & \\
ITA & 8,9 & 13 & 81,8 & 83,1 & $\pm 39,6$ & 1571,8 & 0,09 & 0,26 \\
CUJ & 8,0 & 6 & 112,6 & 114,3 & $\pm 36,3$ & 1318,4 & 0,16 & 0,25 \\
ITB & 6,2 & 15 & 59,9 & 63,0 & $\pm 39,8$ & 1521,2 & 0,61 & 0,35 \\
\hline
\end{tabular}

Em que: $\mathrm{G}=$ área basal das castanheiras $\left(\mathrm{m}^{2} \mathrm{ha}^{-1}\right), \mathrm{N}=$ número de árvores (indivíduos ha ${ }^{-1}$ ); $\mathrm{DAP}=$ diâmetro a altura do peito $(\mathrm{cm})$.

O teste de Kolmogorov-Smirnov, aplicado para comparar as distribuições de frequências diamétricas $(\mathrm{DAP} \geq 10 \mathrm{~cm})$ das populações indicou não haver aderência entre ITA e CUJ $(\mathrm{D}=$ $1,28, p=0,03)$ e CUJ e ITB ( $D=1,32 ; p=0,02)$, porém, as estruturas diamétricas para ITA e ITB aderiram, sendo consideradas semelhantes $(\mathrm{D}=0,686 ; \mathrm{p}=0,367)$.

Exceto em ITB, observou-se maior frequência de indivíduos nas classes intermediárias de diâmetro (Figura 2), corroborando a maioria dos inventários florestais realizados em castanhais nativos (SALOMÃO, 1991; MYERS; NEWTON; MELGAREJO, 2000; ZUIDEMA; BOOT, 2002; PERES et al., 2003; SCOLES; GRIBEL, 2012; 2015). Esta característica é considerada típica para espécies com recrutamento descontínuo associado à ocorrência de eventos irregulares para a promoção da regeneração ou, com a capacidade de crescer mais rapidamente em certas categorias de tamanho, que tornam as classes de maior incremento diamétrico menos abundantes por terem um menor tempo de passagem, ou levarem um menor período de tempo para mudar de classe de diâmetro (MYERS; NEWTON; MELGAREJO, 2000; ZUIDEMA, 2003).

Segundo Myers, Newton e Melgarejo (2000) e Zuidema (2003), a redução observada nas taxas de crescimento diamétrico em árvores de castanheira-do-brasil com DAP maiores que 50 $\mathrm{cm}$ ocasionaria um incremento gradual na abundância de indivíduos adultos e baixa abundância de indivíduos jovens. Este comportamento foi observado por Schöngart et al. (2015) em estudo dendrocronológico com a espécie em castanhal em Oriximiná - PA, ao verificarem taxas máximas de incremento diamétrico em árvores com $\mathrm{DAP}=40,5 \mathrm{~cm}$, com valores decrescentes a partir desta dimensão.

No entanto, este comportamento não pode ser considerado padrão, uma vez que Staudhammer, Wadt e Kainer (2013) ao utilizarem cintas dendrométricas em castanhal no Acre, observaram que o crescimento em área basal foi maior nas árvores entre $30 \mathrm{~cm}$ a $40 \mathrm{~cm}$ de DAP, não sendo observada grande redução de incremento nas maiores classes de diâmetro.

Uma vez que a espécie apresenta dificuldades no estabelecimento da sua regeneração sob o dossel fechado (MYERS; NEWTON; MELGAREJO, 2000), vida longa (SCHÖNGART et al., 2015), que a mortalidade natural esperada é maior em árvores grandes (senescentes) e que as árvores jovens nas menores classes diamétricas por crescerem mais rapidamente, tendem a ser menos frequentes (ZUIDEMA, 2003), presume-se que castanhais mais velhos ou com baixo nível de perturbação antrópica apresentem distribuições do tipo leptocúrticas.

Isto sugere que as populações nas regiões do Itã e Itaúba são mais jovens do que a localizada na região do Cujubim. Reforçam esta hipótese o fato que estas duas populações apresentaram distribuições diamétricas com maior proporção de indivíduos jovens; um menor 
número de indivíduos adultos $(\mathrm{DAP} \geq 40 \mathrm{~cm})$ e, estes, com menores diâmetros. Na comparação com CUJ, as distribuições de frequência em ITA e ITB são mais achatadas com maior grau de curtose, que se deveu a maior densidade de indivíduos nas menores classes diamétricas.

Figura 2 - Estrutura diamétrica das populações de Bertholletia excelsa estudadas.

Figure 2 - Diametric structure of Bertholletia excelsa studied populations.

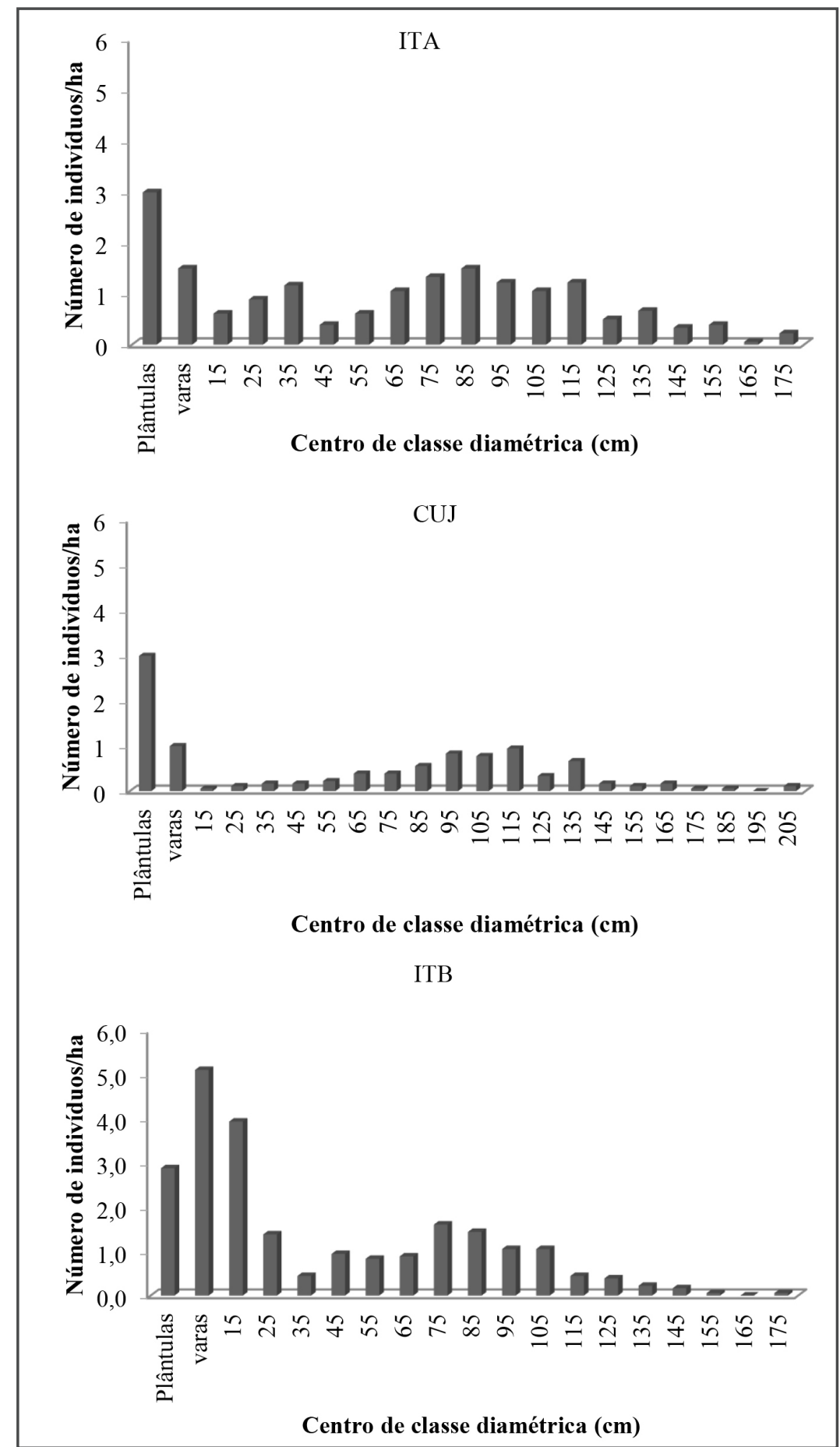

Fonte: Autores (2019) 
Foram observadas 1 a 4 plântulas ha ${ }^{-1}$ e 0 a 10 varetas ha $^{-1}$, o que pode ser considerado muito baixo na comparação com Zuidema e Boot (2002) com 24 e 40 indiv. ha ${ }^{-1}$ (h $\leq 1,4 \mathrm{~m}$ ) e Scoles e Gribel (2015) no Amazonas com 24,8 (h $\leq 1,5 \mathrm{~m}$ ) indiv ha ${ }^{-1}$, e próximos aos observados por Wadt et al. (2008) com 3,8 a 5,8 ( $\mathrm{h} \leq 1,5 \mathrm{~m})$ indiv $\mathrm{ha}^{-1}$ e Souza, Almeida e Amaral (2014) com 5,25 ( $\mathrm{h} \leq 1,0$ $\mathrm{m})$ indiv ha-1. Entre as prováveis razões para estas variações estão as diferenças nos métodos de amostragem, a época de coleta dos dados, a fisionomia florestal e as intervenções antrópicas.

Levantamentos de regeneração natural realizados durante a época chuvosa tendem a observar maior número de regenerantes, devido à germinação ocorrer durante esta época do ano (WADT et al., 2008). Na primeira estação seca, a mortalidade das plântulas pode chegar a $65 \%$ (ZUIDEMA; BOOT, 2002).

Florestas mais abertas e suscetíveis a regimes naturais e antrópicos de distúrbios que levem à formação de clareiras beneficiam a regeneração natural da espécie que é demandante por luz nas fases iniciais de desenvolvimento (TOMAS et al., 2015). Da mesma forma, distúrbios antrópicos como a dispersão realizadas pelos extrativistas e as aberturas do dossel provocadas pela abertura de picadas e pela extração madeireira também podem beneficiar a regeneração (SCOLES; GRIBEL, 2012; MOLL-ROCEK; GILBERT; BROADBENT, 2014).

\section{Relação entre regenerantes, fase de desenvolvimento da floresta e taxa de coleta de sementes}

Em todas as áreas ocorreu a predominância de floresta madura em uma proporção que variou de 54,7\% em CUJ a 90,6\% em ITA. Nas áreas sob a influência do manejo madeireiro (CUJ e ITB), essa proporção diminuiu na medida em que aumentou a ocorrência de clareiras e áreas em reconstrução (Figura 3A). Da área total amostrada para a regeneração natural, $5 \%$ correspondeu a clareiras, $26,4 \%$ a áreas em reconstrução e $68,4 \%$ a floresta madura.

A maior área de clareiras observada em CUJ pode ser atribuída à atividade madeireira recente. Em ITA a clareira existente deveu-se a queda de uma grande árvore e em ITB, apesar de atualmente não existirem clareiras na área experimental, observou-se maior quantidade de áreas em reconstrução, caracterizadas por um grande número de indivíduos do gênero Cecropia.

Foram observadas plântulas em todas as fases de desenvolvimento da floresta e um número de indivíduos por hectare 2,6 vezes maior $\left(\mathrm{x}^{2}=6,27 ; \mathrm{p}=0,043\right)$ nas clareiras (Figura $3 \mathrm{~B}$ ). Não foram observadas diferenças significativas ( $5 \%$ de nível de confiança) entre o número de plântulas, varas e indivíduos jovens por local ( $\mathrm{p}=0,58 ; \mathrm{p}=0,86 ; \mathrm{p}=0,10$, respectivamente). Porém, pode-se admitir um maior número de indivíduos jovens em ITA (Figura 3C) para um nível de confiança de $10 \%$

O maior número de plântulas observado em clareiras concorda com a hipótese de que existe um gradiente de sobrevivência e recrutamento nas diferentes fases de regeneração natural da castanheira-do-brasil, que é maior nas clareiras (ZUIDEMA, 2003; SOUZA; ALMEIDA; AMARAL, 2014). Contudo, não existe relação direta entre a abertura do dossel e o estabelecimento das plântulas da espécie em florestas nativas (MYERS; NEWTON; MELGAREJO, 2000; ZUIDEMA; BOOT, 2002; SCOLES; GRIBEL, 2012; SOUZA; ALMEIDA; AMARAL, 2014), ou seja, durante a fase de plântula, a espécie não apresenta preferência por sítios específicos. A característica de germinar e sobreviver sob dossel aberto e fechado, mas somente se desenvolver em áreas abertas (grandes clareiras e capoeiras) que posteriormente se fecham naturalmente, pode explicar a não significância da relação número de varetas e fase de desenvolvimento da floresta observado neste estudo.

Não houve diferenças significativas $(\mathrm{p}=0,26)$ entre as estatísticas de frutos e suas relações com as plântulas por fase de desenvolvimento da floresta (Tabela 2), porém, observou-se tendência à diminuição na relação entre o número de plântulas e o número de frutos coletados e não coletados entre a clareira e a floresta madura. Esta relação indicou que para cada 100 frutos 
abertos pela fauna, 10 plântulas se estabeleceram em uma clareira e apenas duas na floresta madura. De cada 100 frutos não coletados, sete plântulas se estabeleceram em uma clareira e apenas uma na floresta madura, o que pode explicar o maior número de plântulas observado nas clareiras.

Para avaliar a correlação entre os indicadores de regeneração natural e as variáveis ecológicas também foram adicionados dados de trabalhos de mesma natureza, cujas variáveis foram coletadas de forma a permitir comparações com este estudo (Tabela 3). A intensidade de coleta variou entre $46,3 \%$ a $90 \%$, com as maiores taxas em Itaúba, indicando comparações entre locais com alta intensidade de coleta.

Figura 3 -Área amostrada por local de estudo (A), número de indivíduos por hectare por fase de desenvolvimento da floresta $(B)$ e número de indivíduos por hectare por área monitorada $(C)$. ITA = região do Itã em Caracaraí - RR; CUJ = região do Cujubim em Caracaracaí - RR e ITB = Itaúba - MT.

Figure 3 - Sampled area for the studied sites (A), number of individuals per hectare for forest development phase (B) and number of individuals per hectare per site (C). ITA = Itã region (Caracarai, RR state); CUJ = Cujubim region (Caracarai, RR state) and ITB = Itaúba (MT state).

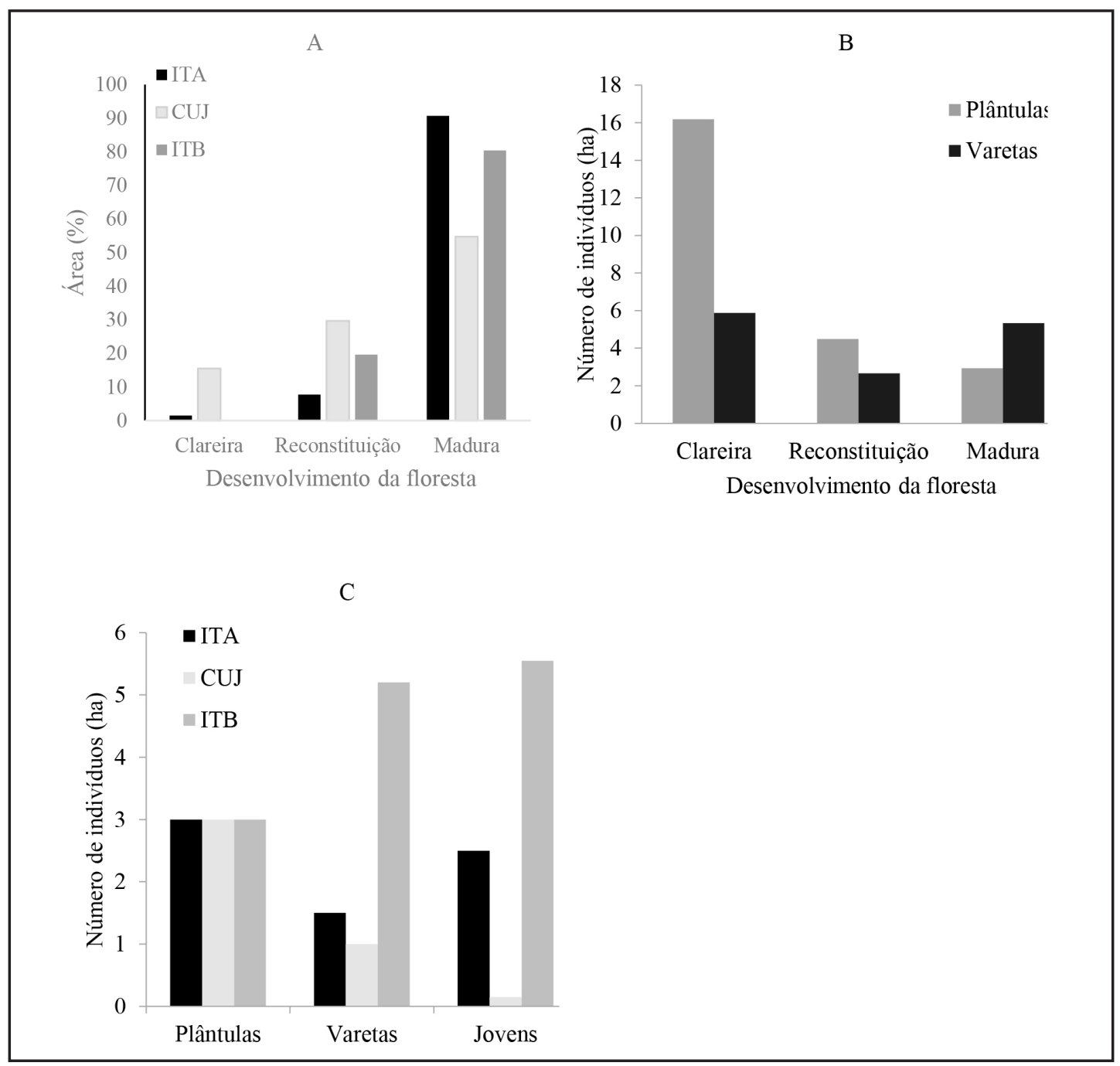

Fonte: Autores (2019) 
Tabela 2 - Frutos de Bertholletia excelsa abertos por roedores e não coletados por fase de desenvolvimento da floresta nas unidades amostrais localizadas na região do Itã e Cujubim em Caracaraí - RR e Itaúba - MT.

Table 2 - Fruits of Bertholletia excelsa opened by rodents and not collected by forest successional stages in the sample units located in Itã and Cujubim region in Caracarai (RR state) and Itaúba (MT state).

\begin{tabular}{|c|c|c|c|c|c|}
\hline Fase de desenvolvimento da floresta & $\mathrm{NF}_{\mathrm{a}}$ & $\mathrm{NF}_{\mathrm{nc}}$ & $\mathrm{NF}_{\mathrm{a}}(\%)$ & $\mathbf{N P} / \mathrm{NF}_{\mathrm{a}}$ & $\mathbf{N P} / \mathrm{NF}_{\mathrm{nc}}$ \\
\hline Clareira & 160 & 225 & 59,4 & 0,10 & 0,07 \\
\hline Reconstrução & 108 & 164 & 66,7 & 0,04 & 0,03 \\
\hline Floresta madura & 143 & 266 & 51,7 & 0,02 & 0,01 \\
\hline
\end{tabular}

Em que: $\mathrm{NF}_{\mathrm{a}}=$ Número de frutos abertos por roedores; $\mathrm{NF}_{\mathrm{nc}}=$ número de frutos não coletados; $\mathrm{NP}=$ número de plântulas.

A atividade da fauna variou entre $13,6 \%$ a $88,8 \%$ sendo maior em ITA. A menor taxa de atividade da fauna em CJU 2 deveu-se a não realização de coletas por dois anos o que implicou uma grande disponibilidade de frutos não absorvidos pela fauna. Ao remover este dado, observouse correlação significativa negativa com a taxa de coleta $(r=-0,90 ; P=0,037)$.

Não foram observadas correlações significativas entre os indicadores de regeneração natural e as variáveis explicativas avaliadas (Tabela 4). Dos frutos deixados na floresta, em média $47 \%$, foram abertos pela fauna, com uma variação entre $19 \%$ em ITB e $70 \%$ em ITA, sendo este percentual menor na área com maior intensidade de coleta.

Tabela 3 - Indicadores de regeneração natural e variáveis ecológicas com potencial explicativo por parcela e área de estudo.

Table 3 - Natural regeneration indicators and explanatory power ecological variables per plot and study sites.

\begin{tabular}{|c|c|c|c|c|c|c|c|c|c|}
\hline Local & NP & NV & NJ & PS & $\mathbf{N}_{\text {adultos }}$ & $\begin{array}{c}\mathbf{N F}_{\mathbf{n c}} \mathbf{l} \\
\mathbf{N}_{\text {adultos }}\end{array}$ & $\begin{array}{c}\mathbf{N P} / \\
\mathbf{N}_{\text {adultos }}\end{array}$ & $\begin{array}{l}\text { IC } \\
(\%) \\
\end{array}$ & $\begin{array}{l}\text { AF } \\
(\%)\end{array}$ \\
\hline Caracarai-RR (ITA1) & 1,0 & 3,0 & 3,0 & 3,48 & 10,3 & 2,7 & 0,1 & 65,7 & 88,8 \\
\hline Caracarai-RR (ITA2) & 5,0 & 0,0 & 2,0 & 2,84 & 11,2 & 18,9 & 0,4 & 50,0 & 61,8 \\
\hline Caracarai-RR (CUJ1) & 2,0 & 1,0 & 0,1 & 18,5 & 5,4 & 23,3 & 0,4 & 78,5 & 51,9 \\
\hline Caracarai-RR (CUJ2) & 4,0 & 1,4 & 0,2 & 14,4 & 5,9 & 174,3 & 0,7 & 46,3 & 13,6 \\
\hline Itaúba-MT (ITB1) & 2,0 & 0,4 & 3,1 & 12,3 & 8,0 & 2,15 & 0,3 & 90,0 & 17,6 \\
\hline Itaúba-MT (ITB2) & 4,0 & 10 & 8,0 & 8,3 & 9,9 & 1,0 & 0,4 & 88,6 & 20,5 \\
\hline Filipinas-AC ${ }^{(1)}$ & 5,8 & 1,8 & 2,3 & N.A & 3,7 & 10,8 & 1,6 & 48,4 & 48,9 \\
\hline Cachoeira-AC ${ }^{(1)}$ & 3,2 & 2,0 & 3,3 & N.A & 6,6 & 10,7 & 0,5 & 56,5 & 47,7 \\
\hline Pindamonhangaba-AC ${ }^{(1)}$ & 5,0 & 3,1 & 2,8 & N.A & 5,9 & 6,3 & 0,8 & 63,3 & 49,8 \\
\hline Vale do Rio Trombetas-PA $(2)$ & 4,8 & 1,0 & 0,5 & N.A & 6,3 & N.A & 0,8 & N.C & N.C \\
\hline
\end{tabular}

Continua... 
Tabela 3 - Conclusão...

Table 3 - Conclusion...

\begin{tabular}{lccccccccc}
\hline \multicolumn{1}{c}{ Local } & NP & NV & NJ & PS & $\mathbf{N}_{\text {adultos }}$ & $\begin{array}{c}\mathbf{N F}_{\mathbf{n c}} / \\
\mathbf{N}_{\text {adultos }}\end{array}$ & $\begin{array}{c}\mathbf{N P} / \\
\mathbf{N}_{\text {adultos }}\end{array}$ & $\begin{array}{c}\mathbf{I C} \\
(\mathbf{\%})\end{array}$ & $\begin{array}{c}\mathbf{A F} \\
\mathbf{( \% )}\end{array}$ \\
\hline Manicoré-AM $^{(3)}$ & 24,8 & 4,4 & 3,2 & N.A & 9,3 & N.A & 2,7 & N.C & N.C \\
Flona Caxiuana-PA $^{(4)}$ & 5,3 & N.C & 11,2 & N.A & 13,7 & N.A & 0,4 & N.C & N.A \\
\hline
\end{tabular}

Em que: $\mathrm{NP}=$ número de plântulas (ha); $\mathrm{NV}=$ Número de varetas (ha); $\mathrm{NJ}=$ número de castanheiras jovens $(\mathrm{DAP} \leq 40 \mathrm{~cm})$ por hectare; $\mathrm{PS}=$ produção média de sementes por árvore adulta; $\mathrm{NF}_{\mathrm{nc}}=$ número de frutos não coletados; $\mathrm{N}_{\mathrm{p}}=$ Número de árvores produtivas; $\mathrm{IC}=$ intensidade de coleta em porcentagem; $\mathrm{AF}=$ atividade da fauna em porcentagem; N.A = Não avaliado; N.C= dado coletado de forma a não permitir comparação com este estudo (1) Fonte: Wadt et al. (2008); (2) Fonte: Scoles e Gribel (2012); (3) Fonte: Scoles e Gribel (2015); (4) Fonte: Souza, Almeida e Amaral (2014).

A relação número de plântulas por número de frutos abertos por roedores $\left(\mathrm{NP} / \mathrm{NF}_{\mathrm{a}}\right)$ variou entre $2 \%$ a $10 \%$, estando próxima à observada por Wadt et al. (2008) no Acre, que variou entre 3\% a $7 \%$, e, ambos, muito inferiores aos observados por Zuidema e Boot (2002) em Riberalta e El Sena no norte da Bolívia, com 0,82 a 3 plântulas por fruto aberto, ou seja $82 \%$ a $300 \%$. As razões para estas variações na germinação e estabelecimento das plântulas entre regiões ainda são desconhecidas pela ciência, mas podem estar relacionadas com a época de coleta dos dados (WADT; KAINER; GOMES-SILVA, 2005), ao comportamento da fauna em relação a taxa de coleta (FORGET, 1996) e as características biogeográficas

Algumas regiões da Amazônia podem apresentar características favoráveis à regeneração natural da espécie. Regiões mais secas e a de transição floresta-savana no sudeste da Amazônia estão sujeitas a maiores distúrbios (aberturas de dossel), ocasionados pela interação da seca com os incêndios florestais (SCOLES; GRIBEL, 2012).

\section{Tabela 4 - Correlação (r) e nível de significância (p) entre os indicadores de regeneração natural de Bertholletia excelsa e as variáveis ecológicas.}

Table 4 - Correlation ( $\mathrm{r}$ ) and significance level ( $\mathrm{p}$ ) between natural indicators of Bertholletia excelsa regeneration and ecological variables.

\begin{tabular}{lcccccc}
\hline \multirow{2}{*}{ Variável } & \multicolumn{2}{c}{ Plântulas $(\mathbf{h a})$} & \multicolumn{2}{c}{ Varetas $(\mathbf{h a})$} & \multicolumn{2}{c}{ Jovens $(\mathbf{h a})$} \\
\cline { 2 - 7 } & $\mathbf{r}$ & $\mathbf{p}$ & $\mathbf{r}$ & $\mathbf{p}$ & $\mathbf{r}$ & $\mathbf{p}$ \\
\hline $\mathbf{P S}$ & $-0,265$ & 0,612 & 0,116 & 0,827 & $-0,486$ & 0,329 \\
$\mathbf{N F}_{\text {nc }} / \mathbf{N}_{\text {adultos }}$ & 0,245 & 0,526 & $-0,544$ & 0,130 & $-0,833$ & $0,002^{*}$ \\
Taxa de coleta (\%) & $-0,599$ & 0,088 & 0,201 & 0,604 & 0,483 & 0,187 \\
Atividade da Fauna (\%) & $-0,093$ & 0,812 & 0,033 & 0,932 & $-0,250$ & 0,516 \\
$\mathbf{N}_{\text {adultos }}$ & 0,025 & 0,939 & 0,087 & 0,798 & & \\
\hline
\end{tabular}

Em que: $\mathrm{PS}=$ produção média de sementes por árvore adulta; $\mathrm{NF}_{\mathrm{nc}}=$ número de frutos não coletados $\mathrm{N}_{\text {adultos }}=$ número de castanheiras adultas $(\mathrm{DAP} \geq 40 \mathrm{~cm})$.

A relação número de varetas e número de plântulas indica o percentual de estabelecimento da regeneração em castanhais nativos, uma vez que, ao atingirem alturas maiores do que 1,3 m, a probabilidade de mortalidade diminui para próximo de zero (ZUIDEMA, 2003).

Com base nos resultados obtidos neste estudo e os trabalhos de Wadt et al. (2008), 
Scoles e Gribel (2012; 2015) e Souza, Almeida e Amaral (2014) observou-se que valores para esta relação próximos ou acima de 1 indicam ausência ou níveis muito baixos de regeneração natural estabelecida. Em áreas com bom estabelecimento da regeneração observaram-se valores entre 0,17 a 0,33 indicando que entre $17 \%$ a $33 \%$ das plântulas conseguem se estabelecer e atingir o estágio de varetas.

A castanheira dispersa seus frutos anualmente, de forma sincrônica, a partir do início das chuvas (FAUSTINO; EVANGELISTA; WADT, 2014; TONINI et al., 2014) por um período que varia de três a seis meses, dependendo da região (CAMPOS et al., 2013; TONINI et al., 2014,) porém, a maioria dos frutos caem em um curto período de tempo, em até 50 dias (FAUSTINO; EVANGELISTA; WADT, 2014).

Esta característica possibilita o acesso aos frutos e sementes pela fauna até o momento da coleta que, normalmente, é feita somente no final do período de queda dos frutos por questões de segurança. O percentual de frutos abertos pela fauna varia entre locais, com percentuais de $29 \%$ até $90 \%$, conforme observado por Zuidema e Boot (2002) e Wadt et al. (2008) em florestas na Bolívia e no estado do Acre, respectivamente.

Neste estudo, a diferença entre a produção de frutos colhidos e não colhidos foi em média de $30,1 \%$ variando entre $10 \%$ a $50 \%$, indicando alto percentual de frutos levados pela fauna e/ou não encontrados pelos coletores.

Não houve correlação significativa entre o número de plântulas e a atividade da fauna, indicando que o estabelecimento das plântulas não dependeu diretamente da dispersão natural, o que também foi observado por Scoles e Gribel (2012;2015).

A não significância para a correlação entre o número de plântulas e a produção de sementes média por árvore adulta indica que a regeneração natural pode não depender diretamente da produção de frutos e da densidade de indivíduos adultos, não corroborando os resultados obtidos por Cotta et al. (2008), Wadt et al. (2008) e Scoles e Gribel (2015). Uma provável explicação seriam diferenças no número de dispersores entre os diferentes sítios, variável que não foi avaliada nos estudos de regeneração.

Corroborando com Zuidema e Boot (2002), Wadt et al. (2008) e Scoles e Gribel (2012; 2015), e discordando de Peres et al. (2003), não foram observadas correlações significativas entre os indicadores de regeneração natural e a taxa de coleta. Pelo modelo de Peres et al. (2003), a variação no número de árvores juvenis observadas neste estudo $(10,1 \%$ a $39,9 \%)$ classificariam as áreas como de coleta leve. No entanto, o maior número de juvenis $(39,9 \%)$ foi observado em ITB que apresentou a maior intensidade de coleta (89,3\%). Portanto, eventos passados como a abertura de clareiras propiciadas pela exploração madeireira podem ter tido maior impacto no estabelecimento e desenvolvimento da regeneração natural.

Esta hipótese pode ser fundamentada com base nos resultados obtidos por Moll-Rocek, Gilbert e Broadbent (2014) e Schöngardt et al. (2015), ao verificarem correlação positiva entre as clareiras abertas pela exploração madeireira e a densidade de regenerantes de castanheira-dobrasil, que podem crescer a uma taxa que permite supor um provável efeito da abertura do dossel sobre a estrutura diamétrica atualmente observada em Itaúba.

Schöngardt et al. (2015) com base em dados de dendrocronologia, calcularam o tempo médio de passagem diamétrica para atingir $10 \mathrm{~cm}$ ao DAP de 27 $\pm 10,1$ anos. Em áreas abertas e clareiras naturais o incremento médio anual em altura e diâmetro da castanheira-do-brasil tem variado entre $0,14 \mathrm{~m}$ a $1,1 \mathrm{~m}$ e $0,1 \mathrm{~cm}$ a $2,05 \mathrm{~cm}$, respectivamente (ZUIDEMA; BOOT, 2002, PEÑA-CLAROS et al., 2002; COTTA et al., 2008; SCOLES; KLEIN; GRIBEL, 2014), indicando que em 18 anos poderiam haver regenerantes com diâmetros variando entre 1,8 a 36,9 cm e alturas entre 2,52 e 19,8 m, o que seria consistente com os indivíduos classificados como jovens (DAP $\leq$ $40 \mathrm{~cm})$ em ITB.

Ao aplicar o modelo proposto por Peres et al. (2013) em castanhais no Amapá, Paiva, Quedes e Funi (2011) observaram que áreas com longo e intenso histórico de coleta seriam classificadas como áreas sem exploração, refutando a hipótese de que castanhais com longo 
histórico de coleta intensiva não apresentam recrutamento e estão fadados a desaparecer. Em castanhais na Bolívia, taxas de coleta de até $93 \%$ do número de frutos também tiveram pouco impacto na regeneração natural e na estrutura dos castanhais (ZUIDEMA; BOOT, 2002).

A hipótese de que a disponibilidade de sementes tem baixo impacto sobre a dinâmica populacional da espécie que é controlada, principalmente, pela longa vida e altas taxas de sobrevivência dos indivíduos reprodutivos parece ser a que mais se adéqua aos dados coletados por diferentes pesquisadores em diferentes locais na Amazônia nos últimos anos. Isto tornaria pouco eficazes, ou mesmo desnecessárias, medidas de restrição de coleta para garantir o estoque de regeneração natural em castanhais nativos, especialmente, em áreas sob influência da exploração madeireira.

\section{Conclusões}

Os castanhais apresentaram estruturas diamétricas diferenciadas, o que pode ser atribuído a diferenças no percentual de indivíduos jovens nas menores classes diamétricas. A maior densidade de regenerantes nas clareiras e a inexistência de correlação significativa entre a taxa de coleta e os indicadores de regeneração natural indicam que a abertura do dossel oriunda da exploração florestal madeireira pode ter sido o fator de maior impacto no estabelecimento da regeneração natural e no rejuvenescimento dos castanhais.

\section{Agradecimentos}

Os autores agradecem ao Conselho Nacional de Desenvolvimento Científico e Tecnológico (Processo 472988/2013-1) pelo auxílio financeiro. A madeireira Vale Verde, ao Grupo DalPai e a José Lopes Primo pelo apoio logístico. A prefeitura Municipal de Itaúba e aos extrativistas pelo apoio na seleção das áreas e coleta dos dados.

\section{Referências}

ALVARES C. A. et al. Köpen's climate classification map for Brazil. Meteorologische Zeitschrift, Stutgard, v. 22, n. 6, p. 711-728, 2013.

BRASIL. Departamento Nacional da Produção Mineral. Projeto RADAMBRASIL. Folha NA.20 Boa Vista e parte das folhas NA 21, Tumucumaque, NB.20 Roraima e NB 21; geologia, geomorfologia, pedologia, vegetação e uso potencial da terra. Rio de Janeiro, 1975. 428 p.

CAMPOS, A. M. et al. Fenologia reprodutiva de Bertholletia excelsa Bonpl., em floresta de terra firme em Mazagão, Amapá. Biota Amazônica, Macapá, v. 3, n. 1, p. 1-8, 2013.

CLEMENT C. R. et al. Origin and domestication of native amazonian crops. Diversity, [s. l.], v. 2, n. 1, p. 72-106, 2010.

CONKLETON, P.; GUARIQUATA, M. R.; ALBORNOZ, M. A. Multiple use forestry planning: timber and Brazil nut management in the community forests of Northern Bolivia. Forest Ecology and Management, Amsterdam, v. 268, p. 49-56, 2011.

COTTA, J. et al. Shifting cultivation effects on Brazil nut (Bertholletia excelsa) regeneration. Forest Ecology and Management, Amsterdam, v. 256, n. 1-2, p. 28-35, 2008.

FAUSTINO, C. L.; EVANGELISTA, J. S.; WADT, L. H. O. Dispersão primária de frutos de castanheira (Bertholletia excelsa Bonpl): importância para o manejo e a conservação da espécie. Boletim do Museu Paraense Emilio Goeldi, Belém, v. 9, n. 2, p. 399-414, 2014.

FORGET, P. M. Removal of seeds of Carapa procera (Meliaceae) by rodents and their fate in 
rainforest in French Guiana. Journal of Tropical Ecology, Cambridge, v. 12, n. 6, p. 751-761, 1996.

IBGE. Manual técnico da vegetação brasileira. Rio de Janeiro, 2012. 271 p.

MOLL-ROCEK, J.; GILBERT, M. E.; BROADBENT, E. N. Brazil nut (Bertholletia excelsa, Lecythidaceae) regeneration in logging gaps in the Peruvian Amazon. International Journal of Forestry Research, [s. l.], v. 1, p. 1-8, 2014.

MYERS, G. P.; NEWTON, A. C.; MELGAREJO, O. The influence of canopy gap size on natural regeneration of Brazil nut (Bertholletia excelsa) in Bolivia. Forest Ecology and Management, Amsterdam, v. 127, n. 1/3, p. 119-128, 2000.

PAIVA, P. M.; QUEDES, M. C. G.; FUNI, C. Brazil nut conservation through shifting cultivation. Forest Ecology and Management, Amsterdam, v. 261, p. 508-514, 2011.

PEÑA-CLAROS, M. et al. Enrichment planting of Bertholletia excelsa in secondary forest in the Bolivain Amazon: effect of cutting line width on survival growth and crown traits. Forest Ecology and Management, Amsterdam, v. 161, n. 1/3, p. 159-168, 2002.

PERES, C. A.; BAIDER, C. Seed dispersal, spatial distribution and population structure of brazil nut trees (Bertholletia excelsa) in Southeastern Amazonia. Journal of Tropical Ecology, Cambridge, v. 13, n. 4, p. 595-616, 1997.

PERES C. A. et al. Demographic threats to the sustainability of Brazil nut exploitation. Science, Washington, v. 1, n. 3, p. 12-14, 2003.

SALOMÃO R. P. Densidade, estrutura e distribuição espacial da castanheira-do-brasil (Bertholletia excelsa $\mathrm{H}$ \& B) em uma floresta tropical de platô na Amazônia Setentrional. Boletim do Museu Paraense Emilio Goeldi, Belém, v. 4, n. 1, p. 11-25, 2009.

SALOMÃO R. P. Estrutura e densidade de Bertholletia excelsa H. \& B (castanha-do-brasil) nas regiões de Carajás e Marabá, estado do Pará. Boletim do Museu Paraense Emílio Goeldi, Belém, v. 1, n. 1, p. 47-68, 1991.

SCHEPARD JUNIOR, G. H.; RAMIREZ, H. "Made in Brazil" human dispersal of the Brazil nut (Bertholletia excelsa, Lecythidaceae) in Ancient Amazônia. Economy Botany, [s. l.], v. 65, n. 1, p. 44-65, 2011.

SCHÖNGART, J. et al. Age and growth patterns of Brazil nut tres (Bertholletia excelsa Bonpl.) in Amazônia, Brazil. Biotrópica, Whashington, v. 47, n. 5, p. 550-558, 2015.

SCOLES, R.; GRIBEL, R. Human influence on the regeneration of brazil nut tree (Bertholletia excelsa Bonpl., Lecythidaceae) at Capanã Grande Lake, Manicoré, Amazonas, Brazil. Human Ecology, New York, v. 43, p. 843-854, 2015.

SCOLES, R.; GRIBEL, R. The regeneration of Brazil nut trees in relation to nut harvest intensity in the Trombetas River valley of Northern Amazonia, Brazil. Forest Ecology and Management, Amsterdam, v. 265, p. 71-81, 2012.

SCOLES, R.; KLEIN, G. N.; GRIBEL, R. Crescimento e sobrevivência de castanheira (Bertholletia excelsa Bonpl., Lecythidaceae) plantada em diferentes condições de luminosidade após seis anos de plantio na região do rio Trombetas, Orixiná, Pará. Boletim do Museu Paraense Emilio Goeldi, Belém, v. 9, n. 2, p. 321-336, 2014.

SILVA, J. N. M. et al. Diretrizes para instalação e medição de parcelas permanentes em florestas naturais da Amazônia Brasileira. Belém: EMBRAPA Amazônia Oriental, 2005. 68 p.

SOUZA, D. G.; ALMEIDA, S. S.; AMARAL, D. D. Estrutura de uma população manejada de castaneira (Bertholletia excelsa) na Floresta Nacional de Caxiuanã, Pará. Boletim do Museu Paraense Emilio Goeldi, Belem, v. 9, n. 2, p. 353-370, 2014. 
STAUDHAMMER, C. L.; WADT, L. H. O.; KAINER, K. A. Tradeoff in basal área growth and reproduction shift over the lifetime of a long-lived tropical species. Oecologia, Berlin, v. 173, p. 45-57, 2013.

TOMAS, E. et al. Uncovering spatial patterns in the natural and human history of Brazil nut (Bertholletia excelsa) across the Amazon basin. Journal of Biogeography, Oxford, v. 42, p. 13671382, 2015.

TONINI, H. et al. Fenologia, estrutura e produção de sementes em castanhais nativos de Roraima e características socioeconómicas dos extrativistas. Boletim do Museu Paraense Emilio Goeldi, Belem, v. 9, n. 2, p. 399-414, 2014.

TUCKHAUGAASEN, J. M. T. et al. Seed dispersal of the Brazil nut tree (Bertholletia excelsa) byscatter-hoarding rodents in a central Amazonian forest. Journal of Tropical Ecology, Cambridge, v. 26, p. 251-262, 2010.

ZUIDEMA, P. A. Demography and management of the Brazil nut tree (Bertholletia excelsa). Riberalta: PROMAB, 2003.

ZUIDEMA, P. A.; BOOT, R. G. A. Demography of the Brazil nut tree (Bertholletia excelsa) in the bolivian amazon: impact of seed extraction on recruitment and population dynamics. Journal of Tropical Ecology, Cambridge, v. 18, n. 1, p. 1-31, 2002.

WADT, L. H. O.; KAINER, K. A.; GOMES-SILVA, D. A. P. Population structure and nut yield of a Bertholletia excelsa stand in Southwestern Amazonia. Forest Ecology and Management, Amsterdam, v. 211, n. 3, p. 371-384, 2005.

WADT, L. H. O. et al. Sustainable forest use in Brazilian extractive reserves: natural regeneration of Brazil nut in exploited populations. Biological Conservation, Essex, v.141, n.1, p. 332-346, 2008. 